\title{
Optionen zur Weiterentwicklung von Anpassungsstrategien
}

Petra Mahrenholz, Jörg Knieling, Andrea Knierim, Grit Martinez, Heike Molitor, Sonja Schlipf

33.1 Ansätze für eine strategische Weiterentwicklung von Anpassung - 336

33.1.1 Inkrementelle und transformative Ansätze für Anpassungsmaßnahmen - 336

33.1.2 Anpassung an den Klimawandel durch governance - 336

33.1.3 Partizipation - 338

33.1.4 Kommunikation - 339

33.2 Anpassung als soziokultureller Wandel - 340

33.3 Kurz gesagt -341

Literatur - 341 
Die gegenwärtigen Trends globaler Treibhausgasemissionen und Klimaprojektionen legen schwerwiegende und weitreichende $\mathrm{Zu}$ kunftsrisiken nahe (Teil I und II, Kap. 31), die eine nachhaltige Entwicklung aller Gesellschaften ernsthaft gefährden. Dabei verstärkt der Klimawandel Risiken wie Erosion, Luft- und Gewässerverschmutzung, Armut und Artensterben. Gleichzeitig sind es soziale, politische und ökonomische Prozesse, Verhältnisse und Strukturen, die für den Klimawandel und die resultierenden gesellschaftlichen Probleme ursächlich sind (Brunnengräber und Dietz 2013). Minderungs- und Anpassungsaktivitäten - wenn gut geplant und umgesetzt (Checkliste in UBA 2013) - können eine nachhaltige Entwicklung fördern und Entwicklungspfade eröffnen, die eine "große Transformation“ ermöglichen (IPCC 2014; Kap. 31). Hierzu wäre - in Erweiterung und mit fließenden Übergängen von inkrementeller Anpassung - eine transformative Anpassung an den Klimawandel erforderlich. Letztere beinhaltet radikale Änderungen, bei denen sich die beeinflussten sozialen oder Umweltsysteme hin zu völlig neuen Mustern, Dynamiken oder Orten bewegen (Definitionen s. Park et al. 2012; Kates et al. 2012; EEA 2013). Das zentrale Ziel inkrementeller Anpassung hingegen ist, Wesen und Unversehrtheit eines Systems oder Prozesses in seinem bisherigen Ansatz zu erhalten (Park et al. 2012; Schipper 2007; Kates et al. 2012; Marshall et al. 2012) und den bestehenden sozialen und kulturellen Ordnungsrahmen nicht zu verändern (Pelling 2011). Inkrementelle Anpassung beinhaltet meist eine Verstärkung von Aktionen, die üblicherweise ergriffen werden, um Verluste durch Klimavariabilität bzw. extreme Wetterereignisse zu mindern oder entsprechende Gewinne zu erhöhen, wie bestehende Deiche zu verstärken, Frühwarnsysteme zu modifizieren oder die Wasserversorgung durch weitere Sparmaßnahmen oder größere Reservoire zu verbessern (EEA 2013; vgl. auch $>$ Kap. 32). Dies dürfte eine angemessene Antwort auf geringe oder moderate Klimarisiken sein. Bei schwerwiegenden und weitreichenden Risiken reicht es nicht, neue Lösungen in überholte Strukturen zu integrieren. Hier sind zusätzlich vorausschauende, transformative Anpassungsaktivitäten erforderlich (Kates et al. 2012), die zugrunde liegende Strukturen und Rahmenbedingungen transformieren und mit sozialen Innovationen einhergehen (Beck et al. 2013). Letzteres schließt auch einen Wandel von Werten und Normen ein. Anpassung sollte deshalb auch als Teil eines übergeordneten Transformationsprozesses aufgefasst werden, der gleichermaßen sozial-ökologische Ungerechtigkeiten abbaut und Demokratie vertieft (Brunnengräber und Dietz 2013).

\subsection{Ansätze für eine strategische Weiterentwicklung von Anpassung}

\subsubsection{Inkrementelle und transformative Ansätze für Anpassungsmaßnahmen}

IPCC (2014) nennt Beispiele für Anpassung, die bereits inkrementelle und transformative Elemente enthalten und in eine Weiterentwicklung von Anpassungsstrategien einbezogen werden sollten, so etwa Baustandards oder gesetzlich festgelegte Risikogebiete. Aus Vorsorgegründen kommt hier zur technisch-öko- nomischen Innovation hinzu, dass soziale Praktiken verändert werden, die neue Muster, Dynamiken und Verortungen anstoßen. Vorschläge zur Anpassung, die in diese Kategorie fallen, sind z. B. die Diversifizierung des betrieblichen Produktionsprogramms in der Landwirtschaft, eine neue Kultur- und Sortenauswahl oder veränderte Anbaugebiete sowie die Nutzung von Grauwasser ( Kap. 18). Ein Vorschlag für Verhaltensänderungen wäre beispielsweise eine eingeschränkte Wasserentnahme in Zeiten der Knappheit. Eine eingeschränkte Bebauung oder gar der komplette Siedlungsrückzug aus (Hochwasser-)Risikogebieten, Deichrückverlegungen oder auch die Wiederherstellung natürlicher Retentionsräume und die Erhöhung der Infiltrationskapazität - z. B. durch Auenrenaturierung oder Änderung der Landnutzung gehören zu den transformativen Anpassungsansätzen, die durch Orts- oder Aktivitätsveränderungen gekennzeichnet sind.

Umfassendere transformative Anpassungsansätze sollten Veränderungsprozesse einleiten, die auch gleichzeitig bestehende soziale Ungerechtigkeiten beseitigen (Pelling 2011). Hier kommt eine andere Auffassung des Zieles von nachhaltiger Entwicklung zum Ausdruck: Zur Reduktion des ökologischen Fußabdrucks kommt die ökologische Gerechtigkeit - meist global und intergenerational - hinzu, die sich beispielsweise im frei zugänglichen Anstieg der Lebensqualität für jeden ausdrückt. Beispielsweise zählt IPCC hierzu die Einführung einer medizinischen Grundversorgung für alle (IPCC 2014). Damit wird klar, dass die Visionen des Zielzustands und der Entwicklungspfade in diese Zielzustände auch das Ausmaß transformativer Anpassung bestimmen.

Die Umsetzung vorausschauender transformativer Anpassung dürfte nicht einfach sein. Gründe hierfür sind zum einen die Unsicherheiten künftiger Klimarisiken, aber auch der Wirkspektren von Anpassungsaktivitäten. Zum anderen spielen hohe finanzielle und soziale Kosten sowie Pfadabhängigkeiten eine Rolle, die dazu beitragen, existierende Gewohnheiten, Systeme und Politiken beizubehalten. Erfolgsfaktoren, die in der Weiterentwicklung von Anpassungsstrategien genutzt werden sollten, sind die Beteiligung von Akteuren, die Sicherung finanzieller Ressourcen für den Transformationsprozess sowie das Monitoring und die Evaluierung des Fortschritts (EEA 2013). Dass sich transformative Maßnahmen rechnen können, zeigt ein aktuell diskutiertes Beispiel für eine Deichrückverlegung inklusive der Wiederherstellung von Überflutungsflächen aus der Garbe-Niederung in Sachsen-Anhalt nahe der Landesgrenzen zu Niedersachsen und Brandenburg. Die Kosten für die Renaturierung dieser Auenlandschaften werden auf 13,7-18 Mio. Euro geschätzt. Dem stehen in einem Klimawandelszenario mit verstärkten Hochwasserereignissen als Nutzen, der sich hauptsächlich aus der Nährstoffretention, dem verbesserten Hochwasserschutz und einer hohen Wertschätzung des steigenden Artenreichtums ergibt, 29,3-46,5 Mio. Euro gegenüber (Tröltzsch et al. 2012).

\subsubsection{Anpassung an den Klimawandel durch governance}

Neben konkreten Lösungen, etwa in Bereichen wie Regenwasserbewirtschaftung, Bauleit- oder Regionalplanung ( Kap. 32), erfordert die Weiterentwicklung bisheriger Anpassungsstrategien 


\begin{tabular}{|c|c|c|c|c|}
\hline & $\begin{array}{l}\text { Formelle } \\
\text { Instrumente }\end{array}$ & $\begin{array}{l}\text { Informelle } \\
\text { Instrumente }\end{array}$ & $\begin{array}{l}\text { Ökonomische } \\
\text { Instrumente }\end{array}$ & $\begin{array}{l}\text { Organisations- } \\
\text { entwicklung }\end{array}$ \\
\hline Charakter & $\begin{array}{l}\text { Verbindlich und eindeutig } \\
\text { durch Gesetze und } \\
\text { Verordnungen }\end{array}$ & $\begin{array}{l}\text { Überzeugung und } \\
\text { Selbstbindung beteiligter } \\
\text { Akteure durch } \\
\text { Information, Beteiligung } \\
\text { und Kooperation }\end{array}$ & $\begin{array}{l}\text { Preisbasierter } \\
\text { Lenkungsmechanismus nach } \\
\text { dem Verursacher- und } \\
\text { Gemeinlastprinzip }\end{array}$ & $\begin{array}{l}\text { Langfristige Einbindung } \\
\text { verschiedener Akteure in } \\
\text { Entscheidungs- und } \\
\text { Umsetzungsprozesse } \\
\text { durch institutionalisierte } \\
\text { Prozesse }\end{array}$ \\
\hline Beispiele & $\begin{array}{l}\text { Festsetzungen zum } \\
\text { vorbeugenden } \\
\text { Hochwasserschutz und } \\
\text { Siedlungsklima in der } \\
\text { Bauleit- und } \\
\text { Regionalplanung, } \\
\text { Bundesraumordnungs- } \\
\text { planung Klimaanpassung }\end{array}$ & $\begin{array}{l}\text { Partizipative, } \\
\text { handlungsfeldüber- } \\
\text { greifende Klima- } \\
\text { anpassungsstrategien } \\
\text { unter Verwendung von } \\
\text { Szenarien, Leitbildern } \\
\text { und Roadmaps, Selbst- } \\
\text { verpflichtungen, Risiko- } \\
\text { karten, Informations- } \\
\text { kampagnen, } \\
\text { Bildungsarbeit, } \\
\text { Zusammenarbeit mit } \\
\text { Schulen etc. }\end{array}$ & $\begin{array}{l}\text { Gesplittete } \\
\text { Abwassergebühren, } \\
\text { Bodenversiegelungsabgaben, } \\
\text { Flächennutzungssteuern, } \\
\text { Ressourcennutzungsrechte, } \\
\text { Lastenausgleich im } \\
\text { Hochwasserschutz, Förder- } \\
\text { programme, öffentliche } \\
\text { Investitionen, } \\
\text { Zielvereinbarungen im } \\
\text { Hochwasserschutz }\end{array}$ & $\begin{array}{l}\text { Regionale Netzwerke mit } \\
\text { institutionalisierten } \\
\text { Trägerschaften, } \\
\text { Beratungseinrichtungen } \\
\text { zur Vermittlung zwischen } \\
\text { Wissenschaft und Praxis, } \\
\text { Leit- oder Stabsstellen in } \\
\text { öffentlichen und privaten } \\
\text { Einrichtungen }\end{array}$ \\
\hline Ziele & $\begin{array}{l}\text { Rechtliche Verbindlichkeit } \\
\text { durch einen } \\
\text { institutionalisierten } \\
\text { Umsetzungsbezug }\end{array}$ & $\begin{array}{l}\text { Umdenken in politischen } \\
\text { Prozessen zu } \\
\text { anpassungsfähigen } \\
\text { governance-Systemen in } \\
\text { lernfähigen Politikzyklen }\end{array}$ & $\begin{array}{l}\text { Proaktive Steuerung der } \\
\text { Handlungsmöglichkeiten } \\
\text { einzelner Akteure zur } \\
\text { Klimaanpassung, Schaffung } \\
\text { eines gesellschaftlichen } \\
\text { Ausgleichs für mögliche } \\
\text { Folgekosten }\end{array}$ & $\begin{array}{l}\text { Verankerung der } \\
\text { Querschnittsaufgabe } \\
\text { Klimaanpassung in } \\
\text { laufende } \\
\text { Umsetzungsprozesse }\end{array}$ \\
\hline $\begin{array}{l}\text { Steuerungs- } \\
\text { modus für die } \\
\text { Klima- } \\
\text { anpassung }\end{array}$ & $\begin{array}{l}\text { Flexibilisierung durch } \\
\text { Befristungen im Einzelfall } \\
\text { oder } \\
\text { „Experimentierklauseln“ }\end{array}$ & $\begin{array}{l}\text { Ebenen-, handlungsfeld- } \\
\text { und grenzübergreifende } \\
\text { Zusammenarbeit }\end{array}$ & $\begin{array}{l}\text { Erleichterung einzelner } \\
\text { Klimaanpassungsmaßnahmen } \\
\text { durch die Unterstützung } \\
\text { privater Akteure }\end{array}$ & $\begin{array}{l}\text { Verstetigung } \\
\text { angestoßener } \\
\text { Klimaanpassungsprozesse } \\
\text { in lernenden } \\
\text { Organisationsformen }\end{array}$ \\
\hline
\end{tabular}

- Abb. 33.1 Instrumente der Klimawandel-governance: Kursiv und rot markierte Maßnahmen dienen transformativen Prozessen

Innovationen sozialen Handelns öffentlicher und privater Akteure, die durch governance gesteuert werden. Die in $\mathbf{0}$ Abb. 33.1 rot markierten Beispiele können transformative Anpassungsaktivitäten unterstützen (IPCC 2014, Tabelle SPM.1). Verwaltungen sollten diese Palette nutzen, denn sie haben eine strategische Verantwortung für eine Anpassung an den Klimawandel (s. auch DSt 2015).

Governance erhöht die Anpassungskapazität und Resilienz von Städten und Regionen ( Abb. 33.1; Baasch et al. 2012; Fuchs et al. 2011; Vollmer und Birkmann 2012; Birkmann und Fleischhauer 2013). Der Weg hin zu resilienten Städten und Regionen kann Innovationen sozialen Handelns fördern und über eine Transformation zu einer klimaverträglichen Gesellschaft führen ( Kap. 31; WGBU 2011). governance muss Wege finden, wie diese Innovationen sozialen Handelns aus ihren Nischen heraus breitenwirksam werden können. So kann eine koordinierte Zusammenarbeit zwischen öffentlichen und privaten Akteuren eine Transformation zu einer nachhaltigen Gesellschaftsordnung beschleunigen (WGBU 2011). Kment (2010) und Bauriedl et al. (2014) weisen darauf hin, dass in den meisten Fällen noch keine ausreichende handlungsfeldübergreifende und querschnittsbezogene Betrachtung erfolgt ( Kap. 32). Um naturräumliche Zusammenhänge sowie Konflikte und Synergien bei der Weiterentwicklung von Strategien berücksichtigen zu können, sollte eine governance der Anpassung an den Klimawandel grundsätzlich ebenen-, handlungsfeld- und grenzübergreifend wirken (Ritter 2007; Knieling et al. 2011a; Overbeck et al. 2008). Deswegen sollten Ziele und Maßnahmen von Anpassungsstrategien mit anderen Strategien verschnitten werden. So sind Anpassungsstrategien in Deutschland idealerweise $\mathrm{zu}$ verschneiden mit

- der Nationalen Nachhaltigkeitsstrategie,

- der Waldstrategie 2020,

- der Nationalen Strategie zur biologischen Vielfalt,

- der Wasserrahmenrichtlinie und der Hochwasserrisikomanagementrichtlinie,

- der Meeresstrategie-Rahmenrichtlinie und Maritimen Raumordnung/IKZM,

- den Politiken zur Energiewende und Biomassestrategie,

- der Rohstoffstrategie und dem Ressourceneffizienzprogramm,

- dem Bundesverkehrswegeplan,

- der Strategie zum Schutz kritischer Infrastrukturen und

- der Hightech-Strategie. 
Verbindliche, formelle Instrumente werden beispielsweise in der raumbezogenen Fach- und der räumlichen Gesamtplanung eingesetzt (Danielzyk und Knieling 2011). Hier ergibt sich u. a. Regelungsbedarf hinsichtlich des sich verändernden Siedlungsklimas ( Kap. 22) und im Hochwasserschutz ( Kap. 16). In Regionalplänen werden z. B. bereits Vorrang- und Vorbehaltsgebiete für den vorbeugenden Hochwasserschutz sowie Überschwemmungsgebiete festgesetzt (BMVBS 2014; Frommer et al. 2013; ARL 2009). Um der den Klimawandelszenarien immanenten Unsicherheit Rechnung zu tragen, könnten Vorrang- und Vorbehaltsgebiete auch flexibel, beispielsweise durch Befristungen in Einzelfällen oder „Experimentierklauseln“, festgesetzt werden (BMVBS 2014; Frommer et al. 2013). Aber auch für Gebäude und andere Infrastrukturen lässt sich, z. B. durch angepasste oder neue Baunormen, eine zukunftsfähige Nutzung begünstigen.

Weil die Handlungsspielräume formeller Instrumente begrenzt sind (BMVBS 2013; Schlipf et al. 2008), werden informelle Instrumente ergänzt. Diese streben Regelungen durch Information, Beteiligung und Kooperation an (Bischoff et al. 2007). Sie schaffen Problembewusstsein und unterstützen einen Wertewandel (Frommer 2009; Greiving 2008). Durch ihren Einsatz können demnach neue strategische Ausrichtungen vorbereitet werden, die eine Transformation zu einer nachhaltigen Gesellschaft (WGBU 2011) ermöglichen. Hierzu könnten Ansätze der regionalen governance und des Risikomanagements zu einer risk governance zusammengeführt werden (Greiving 2005; Knieling et al. 2011b). Diese Zusammenführung impliziert ein Umdenken in politischen Prozessen zu lern- und anpassungsfähigen governance-Systemen in Politikzyklen ( Kap. 32; Horrocks 2005; Frommer 2010). Dies schließt die Nutzung von Szenarien (Alcamo und Henrichs 2009; Albert et al. 2012; Zimmermann et al. 2013; Hagemeier-Klose et al. 2013), Leitbildern und Roadmaps (z. B. Beuckert et al. 2011) sowie eines geeigneten Monitorings ein, das eine kontinuierliche Anpassung der Strategien an veränderte Rahmenbedingungen ermöglicht. Ein weiterführender Schritt einer Klimaanpassungs-governance wäre, governance-Instrumente zu entwickeln, die auf verschiedenen Entscheidungsebenen (z. B. EU, Bund, Land, Region, Kommune) Unsicherheiten einbeziehen (multilevel-governance, Kap. 32).

Als ökonomische Instrumente (Braun und Giraud 2009; Soltwedel 2005; Jordan et al. 2007; Zürn 2008) gelten Zielvereinbarungen, etwa zum Hochwasserrisikomanagement, die auf kommunaler Ebene zwischen den Akteuren abgeschlossen werden, als zielführend (Müller 2004; Greiving 2008). Sie basieren auf einem Ansatz, der quantifizierte Leistungs- und Wirkungsvorgaben mit der Projektförderung verknüpft, sodass Klimafolgenrisiken in einem bestimmten Umfang in einem festgelegten Zeitraum reduziert werden müssen. Im Rahmen der Umsetzung entsteht dabei Freiraum, sodass kreative Lösungsansätze zum Einsatz kommen können (Knieling et al. 2011b; Greiving 2008).

Die Entwicklung unterstützender Organisationsformen kann dazu beitragen, Bevölkerung, Unternehmen und Verbände langfristig als „Mit-Gestalter“ in gesellschaftliche Strategie-, Entscheidungs- und Umsetzungsprozesse hin zu einer nachhaltigen Gesellschaft einzubinden (Danielzyk und Knieling 2011). Diese Organisationsformen unterscheiden sich u. a. in der Trägerschaft, der Verankerung auf der politischen Ebene und dem Grad der
Eigenständigkeit (Corfee-Morlot et al. 2011; Vogel et al. 2007). Boundary organizations übernehmen Vermittlungsaufgaben zwischen Akteuren aus Wissenschaft, Politik und Verwaltung, Wirtschaft und Zivilgesellschaft und arbeiten als Beratungseinrichtungen, Netzwerke einzelner Fachleute, Beauftragte oder Service-Einrichtungen (Bischoff et al. 2007). Sie sollen Fachwissen und praktische Anwendung integrieren (Corfee-Morlot et al. 2011) und Plattformen zur Kommunikation und Kooperation anbieten, über die andere Instrumente angewendet werden können, etwa Szenario- oder Leitbildprozesse (Fröhlich et al. 2014). $\mathrm{Zu}$ diesem Zweck werden zunehmend Klimadienste und Dienste zur Unterstützung einer Klimawandelanpassung aufgebaut (Bundesregierung 2015). Weil solche Prozesse Wissen generieren und Einstellungen bilden können, um zukunftsfähig im Sinne der Nachhaltigkeit zu handeln, ist zu erwarten, dass künftig stärker informelle Instrumente zur Weiterentwicklung von Anpassungsstrategien genutzt werden.

\subsubsection{Partizipation}

Die aktive Beteiligung unterschiedlicher gesellschaftlicher Gruppen bei der Weiterentwicklung von Anpassungsstrategien an den Klimawandel ist eine politische Notwendigkeit, um innovative und kreative Lösungen zu schaffen, die eine breite Akzeptanz finden können (Giddens 2009). Partizipation umfasst ein breites Spektrum möglicher Einflussnahme auf gesellschaftliche Entscheidungsprozesse, das von Stellungnahmen und der Bereitstellung von Erfahrungswissen über die Beteiligung an Planungsprozessen bis hin zum Aushandeln und Entscheiden über Ressourcenverteilung reichen kann ( Kap. 29; Renn 2012). Entscheidend für die Ausgestaltung von Partizipationsverfahren ist deren Zielsetzung und die Motivlage für Beteiligung (Walk 2013). Motive finden sich im individuellen Bedürfnis nach persönlicher Weiterentwicklung, eigenverantwortlichem Handeln und an Kompetenzentwicklung zur Teilhabe an - verbesserten und demokratischen - Entscheidungs- und Gestaltungsprozessen. Beteiligte wollen ihr Wissen und ihre Erfahrungen sowie Interessen und Argumente berücksichtigt sehen. Auch Beteiligungsverfahren zur Weiterentwicklung von Anpassungsstrategien an den Klimawandel sollten im Rahmen dieser unterschiedlichen Zielsetzungen gestaltet werden. Ausschlaggebend für das Gelingen von Partizipationsverfahren ist das Vorhandensein von echten Entscheidungsspielräumen und von Teilhabeangeboten, die von den Beteiligten als „,reell“ wahrgenommen werden, sowie deren Bereitschaft, ihre Zeit und ihr Wissen einzubringen. Wichtig für partizipative oder auch aktionsorientierte Prozesse zur Weiterentwicklung von Anpassungsstrategien ist eine gemeinsame Wissensbasis (Hohberg 2014).

Bereits durchgeführte Beteiligungsverfahren zur Anpassung an den Klimawandel (Knierim et al. 2013) können Erfolgsfaktoren offenbaren und lassen sich im Hinblick auf den Moment der Partizipation (Situationsanalyse, Planung, Umsetzung, Auswertung) und entsprechend dem Beteiligungsgrad (Information, Beratung, gemeinsame Entscheidung über Ziele, über Arbeitsschritte, über Ressourcenverwendung usw.) differenzieren. Aussagekräftig für die Qualität eines partizipativen Verfahrens ist 


\subsubsection{Kommunikation} len und dem Ausmaß, mit dem die Prozessbeteiligten Einfluss auf dessen Verlauf und Ergebnis nehmen können (Ison 2010). Ausschnitthaft werden hier ein Fall der partizipativen Szenarioentwicklung und ein integrativer, informeller regionaler Planungsprozess vorgestellt.

Zimmermann et al. (2013) zeigen beispielhaft für partizipative Szenarioentwicklungen, wie Vertreter aus Politik, Verwaltung, Bevölkerung und Wissenschaft die aufgrund des Klimawandels erwarteten künftigen Landnutzungsänderungen abschätzten und darauf aufbauend ein gemeinsames Verständnis einer erwünschten Zukunft entwickelten. Die Auswertung zeigt, dass Unterschiede u. a. dadurch bedingt waren, wie intensiv sich die Beteiligten mit den Szenarien auseinandersetzten und inwieweit sie selbst an der Entwicklung der Zukunftsbilder teilgenommen hatten. Dabei war die Wahrnehmung konkreter Betroffenheit in einem kleinräumigen Kontext und für die gebietsnahen Teilnehmer leichter als auf regionaler Ebene. Hier war es auch schwieriger, alle potenziell Betroffenen in das partizipative Verfahren einzubeziehen, sodass zum Teil allgemeiner und abstrakter diskutiert wurde. Weiter weisen die Autoren auf die Notwendigkeit hin, ein solches informelles, am Anfang eines Planungsprozesses stehendes Instrument an einen politischen Entscheidungsprozess zu koppeln, der eine gewisse Verbindlichkeit für die erzielten Ergebnisse schafft.

Im Großraum Dresden wurde ein ,integriertes regionales Klimaanpassungsprogramm (IRKARP) “ in einem informellen, unter breiter Beteiligung öffentlicher Partner organisierten Planungsprozess entwickelt (Hutter und Bohnefeld 2013). Aufgrund der großen thematischen Breite eines solchen Programms wurden mehr als hundert Organisationen aus Wissenschaft, Verwaltung und Wirtschaft einbezogen, um deren jeweilige Kompetenzen, Kenntnisse und Erfahrungen berücksichtigen zu können. Letztendlich beteiligten sich an der Formulierung des IRKARP jedoch überwiegend Wissenschaftler und Vertreter aus Behörden und Verbänden, während politische und zivilgesellschaftliche Akteure sich hier nicht einbrachten. Vor diesem Hintergrund stellen die IRKARP-Autoren fest, dass „eine demokratietheoretische Einordnung (...) der IRKARP-Formulierung noch zu leisten" und der gemeinsame Arbeitsprozess „vermutlich nicht als verhandlungsdemokratisch" zu bezeichnen ist (Hutter und Bohnefeld 2013). Mit Blick auf die hohe Anwendungsrelevanz dieses Falls fordern sie daher, dass eine systematische und methodische Herangehensweise an spannungsreiche Anforderungen weiterhin als Thema in Forschung und Praxis bearbeitet werden sollte. Auch die Fragen, ob und wie an dieser Stelle soziale und institutionelle Innovationsprozesse stattfinden, verdienen eine ausführliche und kritische Auseinandersetzung.

Es liegen nun vielfältige Erfahrungen mit Beteiligungsverfahren in einem breiten inhaltlichen Feld und in unterschiedlichen institutionellen Settings vor, die - und das ist das Neue - systematisch dokumentiert und damit für eine methodische Auswertung zugänglich sind. Damit wird das Zusammenwirken von situativen Einflussfaktoren einerseits und gezieltem methodischem Design andererseits schrittweise besser operationalisierbar und somit die Basis gelegt für einen gezielteren und erfolgreichen Einsatz von Partizipationsverfahren in der Zukunft (Baasch et al. 2013).
Grundlegend in der Kommunikation von Themen, die eine Handlungskonsequenz erfordern, ist einerseits die Erkenntnis, dass eine reine Informationsvermittlung nicht direkt zu der gewünschten Handlung führt. Weder Wissen allein (Hellbrück und Kals 2012) noch allgemeine Einstellungen führen zwangsläufig zu einem gewünschten spezifischen Verhalten. Beispielsweise nutzen Personen mit einem hohen Umweltbewusstsein den öffentlichen Personennahverkehr, fliegen aber trotzdem im Urlaub in ferne Länder. Spezifische Einstellungen korrelieren hingegen sehr viel mehr mit konkretem Handeln: Wenn nach dem alltäglichen Mobilitätsverhalten und dem Reiseverhalten im Urlaub gefragt wird, stimmen Einstellungen und Verhalten gut überein. Andererseits ist der Zusammenhang zwischen einer konkreten Handlung und einer sich daraus entwickelnden Einstellung sehr viel deutlicher als umgekehrt (Schahn und Matthies 2008). Forschungen zu umweltrelevanten Einstellungen im Klimakontext sollten demnach nicht im Allgemeinen verbleiben, sondern nach konkreten, spezifischen Handlungskontexten fragen, um eine seriöse Grundlage für Kommunikationskonzepte zu erhalten.

Strategische Weiterentwicklungen sollten deshalb von einer Kommunikation der Anpassung an den Klimawandel begleitet werden, die Aspekte wie Komplexität, Umgang mit Unsicherheit und (Nicht-)Wissen zentral berücksichtigt. Klimaprojektionen für die Zukunft sind unsicher. Sie sind plausibel, treffen aber nicht sicher ein ( Kap. 2). Hier entsteht ein Dilemma zwischen einem Genauigkeitsanspruch der Wissenschaft und der Forderung nach konkreten Daten als Planungsgrundlage vonseiten der Praxis (Heidenreich et al. 2014). Wissenschaftliche Begrifflichkeiten hemmen hier die Kommunikation über Anpassung an den Klimawandel. Eine zielgruppenspezifische Übersetzung dieser Wissenschaftsbegriffe erleichtert ein Verstehen zwischen den Akteuren der Wissenschaft und der Praxis. Statt von Unsicherheit, das als Nichtwissen, Fehlen von Sicherheit vor Gefahren oder Fehlen von Selbstsicherheit missverstanden werden kann, empfiehlt es sich, von „Bandbreiten“ oder alternativ „Spannweiten“ möglicher Entwicklungen zu sprechen. Ebenso kann der Begriff „Risiko“ als ein mögliches Ereignis mit geringer Auftretenswahrscheinlichkeit fehlinterpretiert werden (Grothmann 2014). Die Frage, welche Detailgenauigkeit wie und an wen zu vermitteln ist, sollte bei der Aufbereitung wissenschaftlicher Ergebnisse am Anfang stehen (Heidenreich et al. 2014). Ein gelungenes Beispiel, das einen gesellschaftlichen Bewusstseins- und Wertewandel im Sinne nachhaltiger Entwicklung befördern kann, findet sich im Konzept "Bildung für nachhaltige Entwicklung" (UNESCO 2014).

Komplexe Zusammenhänge sind mit dem klassischen Sender-Empfänger-Paradigma schwer kommunizierbar. Dialogorientierte und auf eine gewisse Dauer angelegte Interaktionen (Zwei-Wege Kommunikation) sind in diesem Kontext zu bevorzugen (Grothmann 2014). Dies ermöglicht einen wechselseitigen Lern- und Entwicklungsprozess der Beteiligten, in dem neue Erkenntnisse entstehen und der Transformationen begünstigen kann. Wichtig für den Anfang von partizipativen oder auch aktionsorientierten Prozessen zur Weiterentwicklung von Anpassungsstrategien ist eine gemeinsame Wissensbasis (Hohberg 2014). 
Besonders wirkungsvoll sind adressatenspezifische Kommunikationsformate, die einen klaren Lebensweltbezug erkennen lassen. Akteursanalysen helfen, alle relevanten Akteure systematisch zu ermitteln und sie anschließend in den Transfer von neuen Erkenntnissen und daraus folgenden Strategieentwicklungen einzubeziehen (Nutz 2014; Stelljes et al. 2014). Welche Kommunikationsinstrumente hier passend sind, hängt entscheidend vom Wissensstand und vom Interesse bzw. der Funktion des Adressaten ab (z. B. Funktionsträger aus Politik, Wirtschaft und Verwaltung, Multiplikatoren aus dem Bildungs- und Medienbereich, soziale Gruppierungen wie Anwohner oder die allgemeine Öffentlichkeit). So sind Funktionsträger - wie die Erfahrung gezeigt hat - eher an schneller und gezielter Information und weniger an langen wissenschaftlichen Texten interessiert. Ein geeignetes Informationsmedium ist das Internet, das präzise und gezielte Ergebnisse und Zusammenhänge bieten sollte. Online-Diskurse bieten die Möglichkeit, neue Adressaten zu erreichen und eine Beteiligung am Diskurs zu eröffnen. Insbesondere für die allgemeine Öffentlichkeit haben Kurzvideos, Simulationen oder Karten eine unterstützende und anschauliche Wirkung. Da ein Großteil der menschlichen Wahrnehmung und Entscheidungsfindung auf Intuitionen und nicht auf rationalem Abwägen beruht, bieten die Bildsprache und damit verbundene Botschaften ein großes Potenzial, um für ein neues Thema $\mathrm{zu}$ sensibilisieren. Hier können Formate wie Klimanovellen oder Comics neue Vermittlungswege sein (Hohberg 2014; Körner und Lieberum 2014). Auch im Bildungsbereich finden interaktive Konzepte deutliches Interesse (Foos et al. 2014).

Anpassung an den Klimawandel sollte künftig erstens möglichst mit Klimaschutz zusammen kommuniziert werden. Klimaschutz hat sich als Thema gut etabliert, das Thema Anpassung an den Klimawandel weitestgehend (noch) nicht (Körner und Lieberum 2014). Das gilt auch für den Bildungsbereich, in dem allerdings ein deutliches Interesse vorhanden ist (Katz und Molitor 2014a). Erfahrungsgemäß kann das Thema Klimaschutz die Kommunikation über Anpassungsfähigkeit erfolgreich einleiten (Katz und Molitor 2014b). Zweitens kann eine Kommunikation der Anpassung an den Klimawandel Gehör finden, wenn die Kommunikation an aktuelle gesellschaftliche Themen anknüpft, z. B. an Innovation, Steigerung der Wettbewerbsfähigkeit, Standortsicherung, demografischen Wandel und im Bildungskontext an Themen wie Gesundheit, Ernährung, Biodiversität, Kultur und urban gardening (Foos et al. 2014). Klimabildungsgärten - nah am urban gardening - sind ein Beispiel für nachhaltige Entwicklung aus dem Bildungsbereich. Drittens sollten zur Kommunikation „Aufmerksamkeitsfenster“ - wie ein aktuelles Starkregenereignis - genutzt werden, weil zu diesem Zeitpunkt die erfahrbare Betroffenheit und der Handlungsdruck bei Akteuren extrem hoch sind. Dabei sollte kein kausaler Zusammenhang zwischen dem Einzelereignis und der Klimaänderung postuliert werden. Oft werden häufigere und heftigere Extremereignisse nicht als Auswirkungen des Klimawandels wahrgenommen (Schuck-Zöller et al. 2014). In diesem Fall ist es - viertens - sinnvoll, bestehende Instrumente wie Katastrophenschutzpläne zu nutzen, um Erkenntnisse aus der Forschung zur Anpassung an den Klimawandel dort zu integrieren ( Kap. 32).

\subsection{Anpassung als soziokultureller Wandel}

) „Es ist absehbar, dass sich aus naturwissenschaftlichen Forschungsergebnissen allein keine Handlungsstrategien ableiten lassen, wie dem Klimawandel zu begegnen ist. Wie Menschen diesen wahrnehmen, welche Folgen er für sie hat und ob und in welcher Weise sie bereit sind, entsprechende Strategien tatsächlich umzusetzen, hängt stark vom jeweiligen sozialen und kulturellen Umfeld ab“ (BMBF 2009).

Der Einfluss, den historische Ereignisse, kulturelle Traditionen, Werte und lokale Wissensmuster auf die Akzeptanz und Umsetzung von Anpassungsmaßnahmen haben, ist bisher bei der Formulierung von Anpassungsstrategien weitestgehend unberücksichtigt geblieben.

Die Ergebnisse der Forschungsprojekte RADOST, PROGRESS und ANiK, deren Untersuchungsgebiete sich in Gefährdungs- und Attraktivitätspotenzial ähneln (CSC 2013), unterstreichen die Bedeutung der geistes- und sozialwissenschaftlichen Ergebnisse für die künftige Anpassungsforschung sowie Weiterentwicklung von Anpassungsstrategien. Dies geschieht insbesondere vor dem Hintergrund, dass „Kommunen zu den zentralen Akteuren der Anpassung an den Klimawandel gehören. (...) Viele Maßnahmen zur Anpassung müssen mit und in den Kommunen entwickelt und umgesetzt werden" (Bundesregierung 2011; Kap. 32). In Kommunen würden daher Anpassungsmaßnahmen eher akzeptiert, wenn die kulturellen Traditionen und Wertvorstellungen ihrer Einwohner, ihre kommunalen Identitäten und lokalen geschichtlichen Entwicklungen in den technisch-planerischen und partizipativen Anpassungsprozessen berücksichtigt werden. Nur so können sich die Akteure breit mit solchen Maßnahmen identifizieren und sie auch umsetzen.

Lokale historische Ereignisse und Entwicklungslinien, jedoch auch die ehemalige politische Zugehörigkeit der Gemeinden zur Bundesrepublik oder DDR drücken sich oft in einem unterschiedlichen Verständnis von ökologischen und soziokulturellen Zusammenhängen und damit einem unterschiedlichen Umweltverständnis aus (Martinez et al. 2014a). In der Gemeinde Timmendorfer Strand in Schleswig-Holstein z. B. deckten sich die Interessen des Küstenschutzes und der Anpassung an den Klimawandel mit den Wünschen für die touristische Entwicklung. Erklären ließ sich dies mithilfe eines Rückblicks auf die sozioökonomische Entwicklung anlässlich einer Jahrtausendsturmflut. Die Sturmflut von 1872 und die danach beginnende touristische Entwicklung können als Gründungsmythos einer Gemeinde verstanden werden, die aus dem Nichts zu einem angesehenen Kur- und Badeort avancierte. Als Motor dieser Entwicklung war der Tourismus somit seit Anbeginn identitätsstiftend für die Gemeinde. Neben den akkumulierten materiellen Werten hat dies auch die immateriellen Werthaltungen wie unternehmerisches Denken, Investitionen in Kultur und Infrastruktur in der Gemeinde geprägt. So wurde ein Anpassungskonzept umgesetzt, das neben dem Küstenschutz auch aktiv dem Tourismus dient. Ausschlaggebend waren dabei die gute finanzielle Stellung der Gemeinde - und die damit vorhandene hohe Anpassungskapazität - sowie der partizipative Planungsprozess. In der Gemeinde Ummanz in Mecklenburg-Vorpommern hingegen wurde das 
Küstenschutz- und Anpassungskonzept des Landes als Eingriff in die hart erarbeitete Identität und immateriellen Werte verstanden. Denn dort sollten viele küstennahe Flächen, die durch Entwässerung erst bewirtschaftbar geworden waren, nun durch das Anpassungskonzept „geopfert“ werden, das u.a. eine Wiedervernässung nicht besiedelter Fläche vorsieht. Im Unterschied zur Gemeinde Timmendorfer Strand kann sich Ummanz die teurere Wunschoption einer Anpassungsmaßnahme in Form einer die gesamte Insel umfassenden Schutzanlage nicht leisten. Obgleich die Gemeinde sich selbst um eine Alternativlösung bemühte, führte dieses partizipative Herangehen nicht zum Erfolg. Die Skepsis gegenüber behördlichen Planungen scheint in Ummanz besonders groß zu sein und ist deutlich geprägt von den örtlichen Erfahrungen aus dem Übergang in ein neues politisch-ökonomisches System nach 1990. Daher ist die Vertrauensbildung schwierig, und Anpassungsmaßnahmen müssen unter Berücksichtigung der Wertvorstellungen der Akteure detailliert erörtert werden (Martinez et al. 2014b).

In Rostock und Lübeck führten differierende städtebauliche Entwicklungen und historische Erfahrungen im Umgang mit Sturmfluten zu unterschiedlichen Vulnerabilitätswahrnehmungen und Resilienzbildungen. Insbesondere sozioökonomische Pfadabhängigkeiten - wie im Fall Rostocks, einer vergleichsweise einkommensschwachen Region - prägen die unterschiedlichen Vorstellungen davon, was als Anpassung machbar und als Hochwasserschutz nötig ist. In den Diskursen in Lübeck wird beispielsweise häufig an frühere Sturmfluten erinnert, denen man langjährig und erfolgreich trotzen konnte, während man in Rostock mit dem Klimawandel die große Hoffnung verbindet, dass durch wärmere Sommer der Tourismus boomen wird und die Stadt dadurch wirtschaftliche Probleme überwinden kann (Heimann und Christmann 2013). Die Motivation zur Anpassung in Lübeck rührt insbesondere aus dem historischen Erbe der Hansestadt her, während in Rostock die Hoffnung auf einen Zugewinn in der Tourismusbranche den Diskurs über Anpassungsmaßnahmen treibt.

Im deutschsprachigen Alpenraum hingegen drückt das Naturgefahrenverständnis und -management der lokalen Akteure ein historisch gewachsenes Vertrauen in ein staatlich-professionell organisiertes Naturgefahrenmanagement aus. Lokale Akteure sehen daher eigenverantwortliches Agieren oft als weniger notwendig an (Kruse und Wesely 2013). Insofern müssten hier Anpassungsmaßnahmen besonders von staatlicher Seite koordiniert und kommuniziert werden, da dies besser mit den Wertvorstellungen der lokalen Akteure korrespondiert. Strategisch sollten hier kommunale Eigenverantwortung und Risikokultur vor dem Hintergrund der zunehmenden eigenen Betroffenheit gefördert werden.

Anpassungsstrategien und -maßnahmen sollten, wie vorstehende Beispiele zeigen, stets aus den jeweiligen Entwicklungstraditionen heraus mit Bezug auf geschichtliche Kontexte sowie lokale Interessen, Werte und Haltungen entwickelt werden. Diese lokalen Gegebenheiten prägen die Identität von Kommunen und Städten und damit auch ihre Fähigkeit zu bestimmten Lösungen entscheidend mit. Werte befinden sich in einem ständigen co-evolutionären Prozess mit der sozioökonomischen Entwicklung von Kommunen, auf den wiederum das politische
Umfeld rahmengebend wirkt. In strategischen Weiterentwicklungen können deshalb Anpassungsbeispiele eher dort erfolgreich übertragen werden, wo sich die betroffenen Kommunen und Städte auch in Werthaltungen und Mentalitäten ähnlich sind (Martinez et al. 2014b).

\subsection{Kurz gesagt}

Anpassung an den Klimawandel kann zur nachhaltigen Entwicklung beitragen, insbesondere wenn sie mit sozialen Innovationen einhergeht. Erste Beispiele - wie etwa Nutzungsbeschränkungen durch gesetzliche Festlegungen - werden bereits diskutiert und sollten verstärkt in strategische Weiterentwicklungen einfließen. Diese transformativen Ansätze zur Anpassung an den Klimawandel schließen oftmals Verhaltensänderungen ein, die im Angesicht möglicher schwerwiegender klimawandelinduzierter Risiken für Umwelt und Gesellschaft erforderlich werden. Die Steuerung mithilfe von Rechts-, ökonomischen und zunehmend auch informellen Instrumenten wie Information und Partizipation tragen entscheidend zur Weiterentwicklung von Anpassung an den Klimawandel bei. Diese Instrumente helfen, Problembewusstsein zu schaffen, bringen kreative Lösungen hervor und können einen Wertewandel unterstützen. Dies setzt voraus, dass sich die Kommunikation auf konkrete Handlungskontexte bezieht, einen Lebensweltbezug hat, dialogorientiert und auf Dauer angelegt ist sowie in Beteiligungsverfahren echte Entscheidungsspielräume für die Beteiligten vorhanden sind und als „reell“ wahrgenommen werden. Anpassungsmaßnahmen werden nur erfolgreich umgesetzt, wenn historische Ereignisse, kulturelle Traditionen, vorhandene Werte und lokales Wissen in die Transformationsprozesse einbezogen werden. Hilfreich ist, wenn unterstützende Organisationen Akteure und deren Netzwerke langfristig als Mitgestalter in strategische Weiterentwicklungen einbinden. Grundsätzlich sollte governance ebenen-, handlungsfeld- und grenzübergreifend angelegt werden.

\section{Literatur}

Albert C, Zimmermann T, Knieling J, von Haaren C (2012) Social learning can benefit decision-making in landscape planning: Gartow case study on climate change adaptation, Elbe valley biosphere reserve. Landsc Urban Plan 105(4):347-360

Alcamo J, Henrichs T (2009) Towards guidelines for environmental scenario analysis. Alcamo J (Hrsg) Environmental futures. The practice of environmental scenario analysis. Elsevier, Amsterdam, S 13-35

ARL (2009) Klimawandel als Aufgabe der Regionalplanung. Positionspapier aus der ARL 81. Hannover. http://shop.arl-net.de/media/direct/pdf/pospaper_81.pdf. Zugegriffen: 20. Jan. 2014

Baasch S, Bauriedl S, Hafner S, Weidlich S (2012) Klimaanpassung auf regionaler Ebene: Herausforderungen einer regionalen Klimawandel. Governance 70(3):191-201

Baasch S, Gottschick M, Knierim A (2013) Partizipation und Klimawandel - ein Resümee. In: Knierim A, Baasch S, Gottschick M (Hrsg) Partizipation und Klimawandel - Ansprüche, Konzepte und Umsetzung. KLIMZUG, Bd. 1. Oekom, München, S 269-279

Bauriedl S, Baasch S, Görg C (2014) Umgang mit Klimawandelrisiken - Mehr Handlungsfähigkeit durch Regionale Governance? In: Knieling J, Rossna- 
gel A (Hrsg) Governance der Klimaanpassung. Akteure, Organisation und Instrumente für Stadt und Region. KLIMZUG, Bd. 6. Oekom, München

Beck S, Böschen S, Kropp C, Voss M (2013) Jenseits des Anpassungsmanagements. $\mathrm{Zu}$ den Potentialen sozialwissenschaftlicher Klimawandelforschung. GAIA 22(1):8-13

Beuckert S, Brand U, Fichter K, von Gleich A (2011) Leitorientiertes Roadmapping Nordwest 2050 Werkstattbericht Nr. 10. Oldenburg, Bremen

Birkmann J, Fleischhauer M et al (2013) Vulnerabilität von Raumnutzungen, Raumfunktionen und Raumstrukturen. In: Birkmann (Hrsg) Raumentwicklung im Klimawandel, S 44-68

Bischoff A, Selle K, Sinning H (2007) Informieren, Beteiligen, Kooperieren. Kommunikation in Planungsprozessen; eine Übersicht zu Formen, Verfahren und Methoden. Rohn Verlag, Dortmund

BMBF - Bundesministerium für Bildung und Forschung (2009) Soziale Dimensionen von Klimaschutz und Klimawandel. http://söf.org/de/1344.php. Zugegriffen: 27. Jan. 2014

BMVBS - Bundesministerium für Verkehr Bau und Stadtentwicklung (2013) Wie kann Regionalplanung zur Anpassung an den Klimawandel beitragen? Ergebnisbericht des Modellvorhabens der Raumordnung „Raumentwicklungsstrategien zum Klimawandel" (KlimaMORO) Bd. 157. BMVBS, Berlin

BMVBS - Bundesministerium für Verkehr, Bau und Stadtentwicklung (2014) Regionale Fragestellungen - regionale Lösungsansätze. Ergebnisbericht der Vertiefungsphase des Modellvorhabens der Raumordnung „Raumentwicklungsstrategien zum Klimawandel" (KlimaMORO). BMVBS-OnlinePublikation 01/2014. http://www.bbsr.bund.de/BBSR/DE/Veroeffentlichungen/BMVBS/Online/2014/DL_ON012014.pdf?_blob=publicationFile\&v=2

Braun D, Giraud O (2009) Politikinstrumente im Kontext von Staat, Markt und Governance. In: Schubert K, Bandelow N (Hrsg) Lehrbuch der Politikfeldanalyse 2.0. Oldenbourg, München, S 159-187

Brunnengräber A, Dietz K (2013) Transformativ, politisch und normativ: für eine Re-Politisierung der Anpassungsforschung. GAIA 22(4):224-227

Bundesregierung (2011) Aktionsplan Anpassung der Deutschen Anpassungsstrategie an den Klimawandel. Deutsche Bundesregierung, Berlin. http:// www.bmub.bund.de/fileadmin/bmu-import/files/pdfs/allgemein/application/pdf/aktionsplan_anpassung_klimawandel_bf.pdf. Zugegriffen: 2. Febr. 2014

Bundesregierung (2015) Fortschrittsbericht zur Deutschen Anpassungsstrategie an den Klimawandel. Deutsche Bundesregierung, Berlin

Climate Service Center CSC- (2013) Workshops "Anpassung an den Klimawandel in Berg- und Küstenregionen" Transatlantische Dialoge. http:// www.hzg.de/science_and_industrie/klimaberatung/csc_web/039029/ index_0039029.html.de. Zugegriffen: 27. Jan. 2014

Corfee-Morlot J, Cochran I, Hallegatte S, Teasdale PJ (2011) Multilevel risk governance and urban adaptation policy. Clim Chang 104(1):169-197

Danielzyk R, Knieling J (2011) Informelle Planungsansätze. In: Akademie für Raumforschung und Landesplanung (Hrsg) Grundriss der Raumordnung und Raumentwicklung. Verlag der ARL, Hannover, S 473-498

DSt (2015) Starkregen und Sturzfluten in Städten - eine Arbeitshilfe. http:// www.städtetag.info/imperia/md/content/dst/presse/2015/arbeitshilfe_ starkregen_sturzfluten.pdf. Zugegriffen: 29. Mai 2015

EEA (2013) Adaptation in Europe - addressing risks and opportunities from climate change in the context of socio-economic developments. European Environment Agency, Kopenhagen

Foos E, Jahnke J, Aenis T (2014) Herausforderungen partizipativer Programmentwicklung - Beispiel KlimaBildungsGärten in Berlin. In: Beese K, Fekkak M, Katz C, Körner C, Molitor H (Hrsg) Anpassung an regionale Klimafolgen kommunizieren. Konzepte, Herausforderungen und Perspektiven. Klimawandel in Regionen zukunftsfähig gestalten. KLIMZUG, Bd. 2. Oekom, München, S 251-264

Fröhlich J, Knieling J, Kraft T (2014) Informelle Klimawandel-Governance Instrumente der Information, Beteiligung und Kooperation zur Anpassung an den Klimawandel. neopolis working papers: urban and regional studies, Bd. 15. HafenCity Universität Hamburg, Hamburg

Frommer B (2009) Handlungs- und Steuerungsfähigkeit von Städten und Regionen im Klimawandel. Der Beitrag strategischer Planung zur Erarbeitung und Umsetzung regionaler Anpassungsstrategien. Raumforschung und Raumordnung 67(2):128-141
Frommer B (2010) Regionale Anpassungsstrategien an den Klimawandel - Akteure und Prozess. Dissertation, TU Darmstadt, Darmstadt. WAR- Schriftenreihe

Frommer B, Schlipf S, Böhm HR, Janssen G, Sommerfeld P et al (2013) Die Rolle der räumlichen Planung bei der Anpassung an die Folgen des Klimawandels. In: Birkmann J (Hrsg) Raumentwicklung im Klimawandel, S 120-148

Fuchs S, Kuhlicke C, Meyer V (2011) Editorial for the special issue: vulnerability to natural hazards. The challenge of integration. Natural Hazards 58(2):609-619

Giddens A (2009) The politics of climate change. Polity Press, UK

Greiving S (2005) Der rechtliche Umgang mit Risiken aus Natur- und Technikgefahren - von der klassischen Gefahrenabwehr zum Risk Governance? Zeitschrift für Rechtsphilosophie 2:53-61

Greiving S (2008) Hochwasserrisikomanagement zwischen konditional und final programmierter Steuerung. In: Jarass HD (Hrsg) Wechselwirkungen zwischen Raumplanung und Wasserwirtschaft. Neue Vorschriften im Raumordnungsrecht und Wasserrecht Symposium des Zentralinstituts für Raumplanung an der Universität Münster und des Instituts für das Recht der Wasser- und Entsorgungswirtschaft an der Universität Bonn, 30.5.2008. Lexxion. Der Jur. Verl., Berlin, S 124-145

Grothmann T (2014) Handlungsmotivierende Kommunikation von Klimawandelunsicherheiten?! Empfehlungen aus der psychologischen Forschung. In: Beese K, Fekkak M, Katz C, Körner C, Molitor H (Hrsg) Anpassung an regionale Klimafolgen kommunizieren. Konzepte, Herausforderungen und Perspektiven. Klimawandel in Regionen zukunftsfähig gestalten. KLIMZUG, Bd. 2. Oekom, München, S 49-64

Hagemeier-Klose M, Albers M, Richter M, Deppisch S (2013) Szenario-Planung als Instrument einer "klimawandelangepassten“ Stadt- und Regionalplanung - Bausteine der zukünftigen Flächenentwicklung und Szenarienkonstruktion im Stadt-Umland-Raum Rostock. Raumforschung und Raumordung 71(5):413-426

Heidenreich M, Feske N, Hänsel S, Riedel K, Bernhofer C (2014) Zum Umgang mit Daten aus Klimamodellen - Herausforderungen für eine regional integrierte Klimaanpassung. In: Beese K, Fekkak M, Katz C, Körner C, Molitor $\mathrm{H}$ (Hrsg) Anpassung an regionale Klimafolgen kommunizieren. Konzepte, Herausforderungen und Perspektiven. Klimawandel in Regionen zukunftsfähig gestalten. KLIMZUG. Oekom, München, S 265-278

Heimann T, Christmann G (2013) Klimawandel in den deutschen Küstenstädten und -gemeinden. Befunde und Handlungsempfehlungen für Praktiker. http://www.irs-net.de/forschung/forschungsabteilung-3/progress/ progress-abschlussbericht.pdf. Zugegriffen: 27. Jan. 2014

Hellbrück J, Kals E (2012) Umweltpsychologie. Springer, Wiesbaden

Hohberg B (2014) Moderierte Onlinediskussion als Kommunikations- und Beteiligungsinstrument - Kontext Klimawandel und Klimaanpassung. In: Beese K, Fekkak M, Katz C, Körner C, Molitor H (Hrsg) Anpassung an regionale Klimafolgen kommunizieren. Konzepte, Herausforderungen und Perspektiven. Klimawandel in Regionen zukunftsfähig gestalten. KLIMZUG, Bd. 2. Oekom, München, S 321-334

Horrocks L (2005) Objective setting for climate change adaptation policy. www. ukcip.org.uk/wordpress/wp-content/PDFs/Objective_setting.pdf. Zugegriffen: 27. Jan. 2014

Hutter G, Bohnefeld J (2013) Vielfalt und Methode - Über den Umgang mit spannungsreichen Anforderungen beim Formulieren eines Klimaanpassungsprogramms am Beispiel von REGKLAM. In: Knierim A, Baasch S, Gottschick M (Hrsg) Partizipation und Klimawandel - Ansprüche, Konzepte und Umsetzung. KLIMZUG, Bd. 1. Oekom, München, S 151-172

IPCC (2014) Climate change 2014: impacts, adaptation, and vulnerability. In: Field CB, Barros VR, Dokken DJ, Mach KJ, Mastrandrea MD, Bilir TE, Chatterjee M, Ebi KL, Estrada YO, Genova RC, Girma B, Kissel ES, Levy AN, MacCracken S, Mastrandrea PR, White LL (Hrsg) Part A: Global and sectoral aspects. Contribution of Working Group II to the Fifth Assessment Report of the Intergovernmental Panel on Climate Change. Cambridge University Press, Cambridge

Ison R (2010) System practice: how to act in a climate change world. Springer, Wiesbaden

Jordan A, Wurzel RKW, Zito AR (2007) New models of environmental governance. Are "new" environmental policy instruments (NEPIs) supplanting or supplementing traditional tools of government? In: Jacob K, Biermann 
F, Busch PO, Feindt PH (Hrsg) Politik und Umwelt. Verlag für Sozialwissenschaften, Wiesbaden, S 283-298

Kates RW, Travis WN, Wilbanks TJ (2012) Transformational adaptation when incremental adaptations to climate change are insufficient. PNAS 109(19):7156-7161

Katz N, Molitor H (2014a) Klimaanpassung - (k)ein Thema in umweltrelevanten Bildungsorganisationen? In: Beese K, Fekkak M, Katz C, Körner C, Molitor $\mathrm{H}$ (Hrsg) Anpassung an regionale Klimafolgen kommunizieren. Konzepte, Herausforderungen und Perspektiven. Klimawandel in Regionen zukunftsfähig gestalten. KLIMZUG, Bd. 2. Oekom, München, S 195-210

Katz N, Molitor H (2014b) Zusammenführung und Ausblick. In: Beese K, Fekkak M, Katz C, Körner C, Molitor H (Hrsg) Anpassung an regionale Klimafolgen kommunizieren. Konzepte, Herausforderungen und Perspektiven. Klimawandel in Regionen zukunftsfähig gestalten, Bd. 2. Oekom, München, S 410-467

Kment M (2010) Anpassung an den Klimawandel. JZ. Mohr Siebeck Verlag, Tübingen, $\mathrm{S} 62-72$

Knieling J, Fröhlich J, Schaerffer M (2011a) Climate Governance. In: Buchholz F, Frommer B, Böhm HR (Hrsg) Anpassung an den Klimawandel - regional umsetzen! Ansätze zur Climate Adaption Governance unter der Lupe. Oekom, München, S 26-43

Knieling J, Fröhlich J, Greiving S, Kannen A, Morgenstern N, Moss T, Ratter B, Wickel M (2011b) Planerisch-organisatorische Anpassungspotenziale an den Klimawandel. In: Storch H von, Claussen M (Hrsg) Klimabericht für die Metropolregion Hamburg. Springer, Berlin, S 248-256

Knierim A, Gottschick M, Baasch S (2013) Partizipation und Klimawandel - Zur Einleitung. In: Knierim A, Baasch S, Gottschick M (Hrsg) Partizipation und Klimawandel - Ansprüche, Konzepte und Umsetzung. KLIMZUG, Bd. 1. Oekom, München, S 9-18

Körner C, Lieberum A (2014) Instrumente der Anpassungskommunikation in nordwest2050. Evaluation der Online-Medien. In: Beese K, Fekkak M, Katz C, Körner C, Molitor H (Hrsg) Anpassung an regionale Klimafolgen kommunizieren. Konzepte, Herausforderungen und Perspektiven. Klimawandel in Regionen zukunftsfähig gestalten. KLIMZUG, Bd. 2. Oekom, München, S 393-409

Kruse S, Wesely J (2013) Adaptives Naturgefahrenmanagement. Passende Maßnahmen für angepasste Organisationen in Zeiten des Klimawandels. Workshopbericht. http://www.wsl.ch/fe/wisoz/projekte/anik/ANiK_Workshopbericht_Adaptives_Naturgefahrenmanagement_2013_final.pdf. Zugegriffen: 27. Jan. 2014

Marshall NA, Park SE, Adger WN, Brown K, Howden SM (2012) Transformational capacity and the influence of place and identity. Environ Res Letters 7(3):034022

Martinez G, Orbach M, Frick F, Donargo A, Ducklow K, Morison N (2014a) The cultural context of climate change adaptation. Cases from the U.S. East Coast and the German Baltic Sea coast. In: Martinez G, Fröhle P, Meier H-J (Hrsg) Social dimensions of climate change adaptation in coastal regions. KLIMZUG, Bd. 5. Oekom, München, S 85-100

Martinez G, Frick F, Gee K (2014b) Zwei Küstengemeinden im Klimawandel Zum sozioökonomischen und kulturellen Hintergrund von Küstenschutz für Planung, Umsetzung und Transfer von Anpassungsmaßnahmen. In: Beese K, Fekkak M, Katz C, Körner C, Molitor H (Hrsg) Anpassung an regionale Klimafolgen kommunizieren. Konzepte, Herausforderungen und Perspektiven. Klimawandel in Regionen zukunftsfähig gestalten. KLIMZUG, Bd. 2. Oekom, München, S 293-306

Müller B (2004) Neue Planungsformen im Prozess einer nachhaltigen Raumentwicklung unter veränderten Rahmenbedingungen -Plädoyer für eine anreizorientierte Mehrebenensteuerung. In: Müller B, Löb S, Zimmermann K (Hrsg) Steuerung und Planung im Wandel. Festschrift für Dietrich Fürst. Verlag für Sozialwissenschaften, Wiesbaden:, S 161-176

Nutz M (2014) Die Klimaanpassungsstrategie Nordhessen - Kurskorrekturen und Profilschärfung mit Hilfe von Instrumenten der systemischen Organisationsentwicklung. In: Beese K, Fekkak M, Katz C, Körner C, Molitor H (Hrsg) Anpassung an regionale Klimafolgen kommunizieren. Konzepte, Herausforderungen und Perspektiven. Klimawandel in Regionen zukunftsfähig gestalten. KLIMZUG, Bd. 2. Oekom, München, S 153-166
Overbeck G, Hartz A, Fleischhauer M (2008) Ein 10-Punkte-Plan „Klimaanpassung“. Raumentwicklungsstrategien zum Klimawandel im Überblick. Informationen zur Raumentwicklung 6/7:363-380

Park SE, Marshall NA, Jakku E, Dowd AM, Howden SM, Mendham E, Fleming A (2012) Informing adaptation responses to climate change through theories of transformation. Glob Environ Chang 22:115-126

Pelling M (2011) Adaptation to climate change. From resilience to transformation. Routledge, London

Renn O (2012) Öffentlichkeitsbeteiligung - Aktueller Forschungsstand und Folgerungen für die praktische Umsetzung. In: Grünewald U, Bens $\mathrm{H}$, Fischer H, Hüttl RF, Kaiser K, Knierim A (Hrsg) Wasserbezogene Anpassungsmaßnahmen an den Landschafts- und Klimawandel. Schweizerbart, Stuttgart, S 184-193

Ritter EH (2007) Klimawandel - eine Herausforderung für die Raumplanung. Raumforschung und Raumordnung 6:531-538

Schahn J, Matthies E (2008) Moral, Umweltbewusstsein und umweltbewusstes Handeln. In: Lantermann E-D, Linneweber V (Hrsg) Grundlagen, Paradigmen und Methoden der Umweltpsychologie. Verlag für Psychologie, Göttingen

Schipper L (2007) Climate change adaptation and development: exploring the linkages. Working Paper 107. Tyndall Centre for Climate Change Research 107:1-17

Schlipf S, Herlitzius L, Frommer B (2008) Regionale Steuerungspotenziale zur Anpassung an den Klimawandel. Möglichkeiten und Grenzen formeller und informeller Planung. RaumPlanung 137:77-82

Schuck-Zöller S, Bowyer P, Jacob D, Brasseur G (2014) Inter- und transdisziplinäres Arbeiten im Klimaservice. In: Beese K, Fekkak M, Katz C, Körner C, Molitor $\mathrm{H}$ (Hrsg) Anpassung an regionale Klimafolgen kommunizieren. Konzepte, Herausforderungen und Perspektiven. Klimawandel in Regionen zukunftsfähig gestalten. KLIMZUG, Bd. 2. Oekom, München, S 97-114

Soltwedel R (2005) Marktwirtschaftliche Instrumente. In: Akademie für Raumforschung und Landesplanung (Hrsg) Handwörterbuch der Raumordnung. ARL, Hannover, S 625-631

Stelljes N, Knoblauch D, Koerth R, Martinez G (2014) Akteursanalyse und Befragungen in RADOST - Klimaanpassung aus Sicht von Akteuren an der Ostseeküste. In: Beese K, Fekkak M, Katz C, Körner C, Molitor H (Hrsg) Anpassung an regionale Klimafolgen kommunizieren. Konzepte, Herausforderungen und Perspektiven. Klimawandel in Regionen zukunftsfähig gestalten. KLIMZUG, Bd. 2. Oekom, München, S 167-178

Tröltzsch J, Görlach B, Lückge H, Peter M, Sartorious C (2012) Kosten und Nutzen von Anpassungsmaßnahmen an den Klimawandel - Analyse von 28 Anpassungsmaßnahmen in Deutschland. Climate Change Reihe des Umweltbundesamtes 10/2012, Dessau-Roßlau. http://www.uba.de/uba-infomedien/4298.html. Zugegriffen: 20. März 2014

UBA (2013) Handbuch zur Guten Praxis der Anpassung an den Klimawandel. Umweltbundesamt, Dessau-Roßlau. http://www.umweltbundesamt.de/ publikationen/handbuch-zur-guten-praxis-der-anpassung-an-den. Zugegriffen: 20. Apr. 2014

UNESCO (2014) Das Konzept der Gestaltungskompetenz. www.bne-portal.de/ index.php?id=55. Zugegriffen: 24 . Jan. 2014

Vogel C, Moser SC, Kasperson RE, Dabelko GD (2007) Linking vulnerability, adaptation, and resilience science to practice: pathways, players, and partnerships. Glob Environ Chang 17:349-364

Vollmer M, Birkmann J (2012) Indikatoren und Monitoring zur Vulnerabilität und Anpassung an den Klimawandel. In: Birkmann J, Schanze J, Müller P, Stock (Hrsg) Anpassung an den Klimawandel durch räumliche Planung - Grundlagen, Strategien, Instrumente. Akademie für Raumforschung und Landesplanung (ARL), Hannover (http://nbn-resolving.de/urn:nbn:de:0156-73192: 66- 87 Zugegriffen am 25.01.2014)

Walk H (2013) Herausforderungen für eine integrative Perspektive in der sozialwissenschaftlichen Klimafolgenforschung. In: Knierim A, Baasch S, Gottschick M (Hrsg) Partizipation und Klimawandel - Ansprüche, Konzepte und Umsetzung. KLIMZUG, Bd. 1. Oekom, München, S 21-35

WGBU (2011) Welt im Wandel - Gesellschaftsvertrag für eine große Transformation. Eigenverlag, Berlin

Zimmermann T, Fröhlich J, Knieling J, Kunert L (2013) Szenario-Workshops als partizipatives Instrument zur Anpassung an den Klimawandel. In: Knierim A, Baasch S, Gottschick M (Hrsg) (Hrsg) Partizipation und Klimawandel, 
Ansprüche, Konzepte und Umsetzung. KLIMZUG, Bd. 1. Oekom, München, S 237-258

Zürn M (2008) Governance in einer sich wandelnden Welt - eine Zwischenbilanz. In: Schuppert GF, Zürn M (Hrsg) Governance in einer sich wandelnden Welt, 1. Aufl. Politische Vierteljahresschrift: PVS, Sonderheft, Bd. 41. Verlag für Sozialwissenschaften, Wiesbaden, S 553-580

Open Access Dieses Kapitel wird unter der Creative Commons Namensnennung 4.0 International Lizenz (http://creativecommons.org/ licenses/by/4.0/deed.de) veröffentlicht, welche die Nutzung, Vervielfältigung, Bearbeitung, Verbreitung und Wiedergabe in jeglichem Medium und Format erlaubt, sofern Sie den/die ursprünglichen Autor(en) und die Quelle ordnungsgemäß nennen, einen Link zur Creative Commons Lizenz beifügen und angeben, ob Änderungen vorgenommen wurden.

Etwaige Abbildungen oder sonstiges Drittmaterial unterliegen ebenfalls der genannten Creative Commons Lizenz, sofern sich aus der Abbildungslegende oder der Quellreferenz nichts anderes ergibt. Sofern solches Drittmaterial nicht unter der genannten Creative Commons Lizenz steht, ist eine Vervielfältigung, Bearbeitung oder öffentliche Wiedergabe nur mit vorheriger Zustimmung des betreffenden Rechteinhabers oder auf der Grundlage einschlägiger gesetzlicher Erlaubnisvorschriften zulässig. 